Article (refereed)

Cave, Vanessa M.; King, Ruth; Freeman, Stephen N.. 2010 An Integrated Population Model From Constant Effort Bird-Ringing Data. Journal of Agricultural, Biological, and Environmental Statistics, 15 (1). 119-137. 10.1007/s13253-009-0001-2

() Springer Science+Business Media 2010

This version available http://nora.nerc.ac.uk/13625/

NERC has developed NORA to enable users to access research outputs wholly or partially funded by NERC. Copyright and other rights for material on this site are retained by the authors and/or other rights owners. Users should read the terms and conditions of use of this material at http://nora.nerc.ac.uk/policies.html\#access

This document is the author's final manuscript version of the journal article, incorporating any revisions agreed during the peer review process. Some differences between this and the publisher's version remain. You are advised to consult the publisher's version if you wish to cite from this article.

The original publication is available at www.springerlink.com 


\title{
An Integrated Population Model from Constant Effort Bird-Ringing Data
}

\author{
Vanessa M. Cave ${ }^{1 *}$, Ruth King ${ }^{1}$, Stephen N. Freeman ${ }^{2}$
}

\begin{abstract}
Data from annual bird-ringing programmes, in which catch effort is standardised, are routinely used to index abundance, productivity and adult survival. Efficient models have been developed for each. Such monitoring schemes, based on ringing across a number of sites, are perhaps unique in providing this combination of demographic information and make the data particularly amenable to an integrated approach to population modelling. We develop a Bayesian approach and a deterministic population model uniting abundance, productivity and survival. The method is applied to Sedge Warbler Acrocephalus schoenobaenus data from the British Trust for Ornithology's Constant Effort Site scheme. The possibility of "transient" birds needs to be incorporated within this analysis. We demonstrate how current methodology can efficiently be extended to use additional data from multiple within-year recaptures when controlling for transience.
\end{abstract}

Key words: Bayesian approach; Capture-recapture; Constant Effort Site scheme; Emigration; Sedge Warbler (Acrocephalus schoenobaenus); Transients.

\section{INTRODUCTION}

Monitoring the status of wild bird populations at a national (or even higher) level is becoming increasingly common (Sauer, Hines, Gough, Thomas and Peterjohn, 1997;

Van Strien, Pannekoek and Gibbons, 2001). Surveys on this large geographic scale tend

${ }^{1}$ CREEM, University of St Andrews, Buchanan Gardens, St Andrews, Fife KY16 9LZ, UK.

${ }^{2}$ Centre for Ecology and Hydrology, Crowmarsh Giff ord, Wallingford, Oxfordshire OX10 8BB, UK.

* Corresponding author (vanessa@mcs.st-and.ac.uk) 

to require volunteer-based input at a sample of sites selected either according to a formal design or ad hoc by the volunteers themselves. Such projects are usually orientated towards gathering data to estimate either abundance or various demographic parameters (survival, or components of the productivity of young). This information forms the knowledge base that underpins conservation action, for example in the UK the value of abundance indices is illustrated by their use in the establishment of Biodiversity Action Plans (Gregory, Noble and Custance, 2004).

Of increasing interest is the relationship between abundance and the underlying demographic parameters. This relationship is usually expressed mathematically in the form of a matrix-based population model (Caswell, 2001), which serves to relate the abundance in a given year as a function of that in the previous year(s) and the intermediate vital and reproductive rates. Many papers have been published in which abundance and demographic parameters are simultaneously estimated using data from two or more diff erent surveys (Brooks, King and Morgan, 2004; Besbeas and Freeman, 2006; Schaub, Gimenez, Sierro and Artellaz, 2007; Reynolds et al., 2009). Such analyses, which combine diff erent sources of data, are generally referred to as "Integrated Population Models" .

Typically, indices of abundance are derived from annual counts at a series of sites (Underhill and Pry s-Jones, 1994; Peach, Baillie and Balmer, 1998; Fewster, Buckland, Siriwardena, Baillie and Wilson, 2000), and survival estimates from ringing data (Peach,

1993; Siriwardena, Baillie and Wilson, 1999; Brooks, Catchpole and Morgan, 2000). Productivity rates in integrated models have been previously estimated either as a free parameter in the population model (Besbeas, Freeman, Morgan and Catchpole, 2002; Besbeas and Freeman, 2006; King, Brooks, Mazzetta, Freeman and Morgan, 2008), or by including estimates of breeding success (at least on a per nesting attempt basis) from nest record data (Freeman, Robinson, Clark, Griffin and Adams, 2007; Reynolds et al., 2009).

In this paper we describe the fi rst integrated model to adopt a diff erent source of information, namely annual survey data from "Constant E ff ort" ringing programmes, for example the British Trust for Ornithology' s (BTO) Constant Eff ort Site (CES) scheme and the North American Monitoring Avian Productivity and Survivorship (MAPS) pro- 
gram (DeSante, O' Grady and Pyle, 1999). Such schemes provide information on the key

demographic parameters: abundance, productivity and adult survival, making them particularly amenable to an integrated approach to population modelling. In order to obtain estimates for these demographic parameters independent models have been developed. Building upon these existing models, we formulate an integrated model by specifying a meaningful functional relationship between these demographic parameters. This integrated approach has the advantage of producing estimates that are consistent with both the data and, according to the relationship imposed by the underlying population dynamics, with one another. In addition, measures of productivity over an entire season (uniting multiple broods, and allowing for immediate post-fl edging mortality) are available.

Survival rates have long been estimated from the live-recapture type of data gathered in a Constant Eff ort scheme. Indeed, they are particularly valuable for this, although only rates for adult birds tend to be estimable. However the presence of transient birds in the catch data (i.e. non-resident birds observed only once as they pass through the catch site) produces a negative bias in the estimates of survival (Pradel, Hines, Lebreton and Nichols, 1997). We build upon the model of Pradel et al. (1997) to estimate the proportion of transient birds in the sample, making use of specifi c advantages in this respect customarily provided by Constant Eff ort data, and one that can be adopted either in isolation or as part of an integrated analysis.

Despite adult birds exhibiting strong breeding philopatry (Wernham et al., 2002) some permanent emigration from the site of breeding, but within the domain of the population is expected, the extent of which is typically unknown. The integrated model enables both the magnitude of permanent emigration to be assessed and the estimates of adult survival from Constant Eff ort data to be adjusted accordingly.

The high dispersal of juvenile birds usually prevents accurate estimation of survival in the fi rst year of life using Constant Eff ort data on live-recaptures in subsequent ringing sessions. Separate information, from an entirely diff erent data source, can however be obtained from models for recoveries of dead birds, for which there is again a long history (Seber, 1971; Burnham, 1990). For most small passerine species, especially long-distance 
migrants, these data are however too few to provide precise survival estimates when analysed in isolation. Only by integration with other sources of data can juvenile survival be usefully calculated. We show that an integrated approach has an additional advantage; it gives precise inference on juvenile survival even with limited ring-recovery data.

In Section 2 we introduce the methods underlying Constant Eff ort ringing, and the theory underlying an Integrated Population Model for such data. In Section 3 we fi t the models to a set of data describing captures of Sedge Warblers Acrocephalus schoenabaenus under the longest-running such scheme, overseen by the BTO. The paper concludes with discussion in Section 4. Throughout we assume the population monitored is closed, with all emigration and transient movements between breeding sites occurring within the UK.

\section{DATA AND STATISTICAL MODELS}

\section{1 “Constant Effort” Data}

The BTO' s CES scheme is a volunteer ringing programme conducted annually at a large number of sites. Volunteers aim to visit their particular site on twelve separate occasions that span the breeding season (May-August), and use a standardised mist- netting procedure, within and between years, to capture birds. Captures include both juvenile birds (i.e. those born within the current CES season that are a few weeks old at most), and adult birds (i.e. those born in a previous CES season). All birds caught are fi tted with individually identifying aluminium rings. Over 100 sites now provide data to the scheme annually, and from these the scheme routinely monitors 25 species (Baillie et al., 2009). Note that each CES visit is limited to one of 10 11 possible days, and successive visits to the same site are never less than 3 days apart, usually more. More comprehensive descriptions of the history and fi eld procedure are given by numerous sources (Peach et al.,

1998; Robinson, Freeman, Balmer and Grantham, 2007). We consider in detail data for Sedge Warblers, one of the most frequently encountered species under the CES scheme. Studies of Sedge Warblers are particularly interesting as this species is known to be susceptible to environmental change (Peach, Baillie and Underhill, 1991). 
A typical segment of CES data giving the full capture history for an individual bird would be:

A334831 SEDWA AD 301199500011000000000000

A334831 SEDWA AD 30119960001111111000110000

A334831 SEDWA AD 301199790001100100000009

From left to right the columns represent the ring ID, species, age, site of capture, year of capture and the twelve-visit capture history in which " 9" denotes a missed visit, and otherwise, " 1 " denotes capture and " 0 " that the bird was unobserved. For example, Sedge Warbler A334831 was caught as an adult at site 301 in 1995, 1996 and 1997. At site 301, in 1997, mist-netting was not carried out at visits 1 and 12, but for those visits made A334831 was caught only at visits 4 and 6 .

We consider here data for which the full 12 visits are made within each year, $56 \%$ of the total data set. For example, in the above segment of data records from site 301 in 1997 would be excluded. When one or more visits are missed an incomplete capture record results, and the assumption of constant annual eff ort for that year is violated. This lack of constant eff ort requires special treatment, and methods for dealing with missed visits in the separate modelling of survival and abundance are discussed in Cave, Freeman, Brooks and King (2008) and Cave, Freeman, Brooks, King and Balmer (2009).

The full data set of complete capture records contains 192 sites at which Sedge Warblers have been ringed, however many of these have been operated for few years, and/or caught only a few Sedge Warblers. Sites with such sparse data contribute little information to the analyses at the cost of extra site-specifi c parameters. Therefore we work with a slightly reduced data set which contains only those sites with complete capture records for 4 years or more that caught appreciable numbers of Sedge Warblers (at least

10 adults, and at least 10 juveniles over the duration of its operation). This reduced, " best" sites data set contains 71 sites which, between them, contribute $78 \%$ of the total number of captures (Table 1).

[Table 1 about here.] 


\subsection{Estimating Abundance and Productivity}

We are interested in making inference regarding the population of adult birds breeding in the UK, and the numbers of juvenile birds they produce. Strictly, the CES scheme provides data on adult and juvenile birds at the sites surveyed, however these birds can be assumed to be representative of the wider UK population (Peach et al., 1998).

Given that the full complement of 12 visits is carried out at site $\mathrm{g}$ in year $\mathrm{t}$ the numbers of unique adult and juvenile birds caught are denoted by $n_{g t}^{a}$ and $n_{g t}^{j}$ respectively. A natural estimate of the trend in adult abundance follows by assuming that the $n_{g t}^{a}$ are independent, random variables from a Poisson distribution with parameter $\lambda_{g \mathrm{t}}^{\mathrm{a}} \mathrm{We}$ express $\lambda_{\text {gt }}^{a}$ as a log-linear combination of an intercept term, $\beta^{a}$, plus site- and year-specifi $c$ eff ects, sga and $y^{a}$ respectively (Peach et al., 1998; Cave et al., 2009). Mathematically,

$$
\ln \left(\lambda_{\mathrm{gt}}^{\mathrm{a}}\right)=\beta^{\mathrm{a}}+\mathrm{s}_{\mathrm{g}}^{\mathrm{a}}+\mathrm{y}_{\mathrm{t}}^{\mathrm{a}}
$$

An estimated index of adult abundance in year $t$ is given by $A_{t}=\exp \left(y_{t}^{a}\right)$.

Similarly, for juveniles, we assume that the $\mathrm{n}_{\mathrm{gt}}^{\mathrm{j}}$ are independent, random variables from a Poisson $\left(\lambda_{g t}^{\mathbf{j}}\right)$ distribution and form the analogous log-linear model to (1) for $\lambda_{g t}^{\mathbf{j}}$.

$$
\ln \left(\lambda_{g t}^{j}\right)=\beta^{j}+s_{g}^{j}+y_{t}^{j}
$$

An estimated index of juvenile abundance in year $t$ is given by $J_{t}=\exp \left(y_{t}^{j}\right)$.

For identifi ability, the fi rst year eff ect and an arbitrary reference site eff ect in both

(1) and (2) are constrained to zero.

We defi ne the true seasonal productivity in year $\mathrm{t}_{\mathrm{t}} \mathrm{P}_{\mathrm{t}}^{\mathrm{s}}$, as the ratio of juvenile to adult birds in the population. The capture probabilities of adult and juvenile birds are likely to be unequal due to behavioural diff erences, but under the assumption of constant eff ort, these diff erences are expected to remain constant over time (Peach, Buckland and Baillie,

1996). Therefore, we let $k$ denote an unknown positive scaling factor that quantifi es the extent to which juvenile birds are more or less catch-able than adult birds, and write

$$
P_{t}^{s}=k P_{t}=k \frac{J_{t}}{A_{t}}
$$


$\mathrm{P}_{\mathrm{t}}$ (an index of productivity) is a more useful parameter biologically than $\mathrm{J}_{t}$ itself as it adjusts for the numbers of adult birds producing young.

Likelihoods $L_{a}$ and $L_{j}$ under models (1) and (2) are easily formed and fi tted, respectively, to the observations $n_{g t}^{a}$ and $n_{g t}^{j}$. Estimates of the unknown parameters are obtainable either by maximum likelihood (as conventionally) or from their posterior distributions calculated via MCMC techniques, as in the present paper.

Juvenile Sedge Warblers are dependent upon their parents only for up to a fortnight after fl edging (Alker and Redfern, 1996). Then, the adults depart fi rst for pre-migratory feeding grounds in July (Cramp, 1992). Assuming therefore that the Poisson distributions for the adult and juvenile counts are eff ectively independent, it can be readily shown that

$$
n_{g t}^{j} l\left(n_{g t}^{j}+n_{g t}^{a}\right) \sim \operatorname{Binomial}\left(n_{g t}^{j}+n_{g t}^{a}, \theta_{g t}\right) \text { where } \theta_{g t}=\frac{\lambda_{g t}^{j}}{\left(\lambda_{g t}^{j}+\lambda_{g t}^{a}\right)},
$$

the conditional distribution typically specifi ed for juvenile counts (Julliard, Jiguet and Couvet, 2004; Robinson et al., 2007). Note, under the assumption logit $\left(\theta_{g t}\right)=\beta^{p}+s^{p}+y^{p}{ }_{t}$ where $\beta^{p}$ is an intercept term and $s_{g}^{p}$ are $y_{t}^{p}$ are site and year eff ects respectively, it can be readily shown that $P_{t}=\exp \left(y_{t}^{p}\right)$. However, although this Binomial model based on the conditional distribution for $\mathrm{n}_{\mathrm{gt}}^{\mathrm{j}}$ produces analogous productivity indices to those derived from the Poisson models (1) and (2) via equation (3), in the integrated context the Poisson formulation is preferred since here the joint likelihood for adult and juvenile counts is simply the product of their individual likelihoods.

\subsection{Integrated Models for CES Data}

Clearly the adult and juvenile abundance parameters are estimated independently. The integrated approach involves creating a meaningful functional relationship between these parameters and, assuming independence, multiplying $L_{a}$ and $L_{j}$ to form a single joint likelihood.

We assume that all Sedge Warblers breed after their fi rst winter, aged around one year, and every year thereafter (Cramp, 1992). Furthermore, we assume that there is no net immigration or emigration from the UK population. Let $\varphi_{\mathrm{a}, \mathrm{t}}$ and $\varphi_{\mathrm{j}, \mathrm{t}}$ denote the 
probabilities of survival for an adult and a juvenile bird from year $t$ to $t+1$ respectively. We defi ne the deterministic population model for the index of adult abundance as:

$$
\begin{aligned}
A_{t} & =A_{t-1} \varphi_{a, t-1}+k J_{t-1} \varphi_{j, t-} \\
& =A_{t-1} \varphi_{a, t-1}+k A_{t-1} P_{t-1} \varphi_{j, t-1} .
\end{aligned}
$$

This expression gives the abundance index of adult birds in year $t$, presumed to be breeding, as a function of the abundance index, productivity index and survival probabilities of juvenile and adult birds in the previous year (see Freeman and Crick, 2003).

Recursively:

$$
A_{t}=g\left(A_{1}, k, P_{1 \ldots t-1}, \varphi_{a, 1 \ldots t-1}, \varphi_{j, 1 \ldots t-1}\right) .
$$

Substituting (5) into $L_{a}$ we write a joint likelihood for the adult and juvenile counts as:

$$
\begin{aligned}
L_{\text {joint }} & =L_{a} \times L_{j} \\
& =\mathbf{f}\left(n^{\mathrm{a}} \mid \mathrm{A}_{1}, \mathrm{k}, \mathrm{P}_{1 \ldots T-1}, \varphi_{\mathrm{a}, 1 \ldots T-1}, \varphi_{\mathrm{j}, 1 \ldots T-1}, \beta^{\mathrm{a}}, \mathrm{S}_{1 \ldots \mathrm{G}}^{\mathrm{a}}\right) \mathbf{f}\left(\mathrm{n}^{\mathbf{j}} \mid \mathbf{J}_{1 \ldots \mathrm{T}}, \beta^{\mathbf{j}}, \mathrm{s}_{1 \ldots \mathrm{G}}^{\mathrm{j}}\right),
\end{aligned}
$$

where $T$ and $G$ denote the number of study years and sites respectively.

There is no direct information in either the juvenile or adult CES count data to estimate the constant k, instead it is estimated indirectly via (6) and its role in the deterministic population model (4).

Fully time-varying demographic parameters clearly cannot be estimated from (6) alone. Thus we consider additional data, which provide direct information on survival rates, augmenting the joint likelihood (6) by multiplication with further likelihoods for data on recoveries after death of largely diff erent birds from the BTO' s National Ring- Recovery (NRR) database ( $\left.L_{\text {dead }}\right)$, or live-recaptures of birds during the CES itself $\left(L_{\text {live }}\right)$.

\subsection{Ring-Recovery Data}

The likelihood for ring-recovery data in two age classes is well documented (Freeman and Morgan, 1992; Vounatsou and Smith, 1995). However, as is frequently the case in European bird studies, the number of Sedge Warblers ringed in the BTO' $S$ NRR database (Coiff ait et al., 2008) is not available so we adopt a parameter-reduced form, conditional upon the numbers of dead birds recovered (North and Morgan, 1979; Burnham, 1990). 
Annual totals of recoveries in year $\mathbf{t}$ from each ringed cohort $\mathbf{i}$, separately for birds ringed as adults and juveniles, denoted by $D_{i t}^{a}$ and $D_{i t}^{j}$ respectively, form a collection of independent Multinomial distributions. Recovery probabilities of passerines are widely believed to have declined over time (Baillie and Green, 1987). However Sedge Warblers are shortlived birds hence, for example, those ringed in the early years will have died long before lower rates applicable to recent cohorts become prevalent. Thus we adopt the common practice of ignoring temporal variation and assume that the probability a dead bird is recovered remains constant within each cohort. Therefore, the conditional probability of recovery in year $t$ from cohort $i$ is given by the probability of a bird dying in year $t$ divided by the complement of the probability of its still being alive at the end of the recovery period (North and Morgan, 1979). The likelihood for the ring-recovery data, which is simply a product of Multinomials for both juvenile and adult recoveries, is given by

$$
L_{\text {dead }}=\mathbf{f}\left(D^{\mathrm{j}} \mid \varphi_{\mathrm{j}, 1 \ldots \mathrm{T}}, \varphi_{\mathrm{a}, 2, \ldots \mathrm{T}}\right) \times \mathbf{f}\left(\mathrm{D}^{\mathrm{a}} \mid \varphi_{\mathrm{a}, 1 \ldots \mathrm{T}}\right) .
$$

We consider dead recoveries of birds ringed in Britain during the summer months (May-August), only a small number of which were ringed on CES sites. Those few recoveries of birds ringed in the nest are excluded. The BTO' $s$ NRR database contains recoveries of 294 Sedge Warblers ringed as fl edged birds from 1987 to 2005, of which 257 were ringed as juveniles.

\subsection{Capture-Recapture Data}

The capture-recapture data consist of live resighting histories for individual birds over the study period and fall into the category of mark-recapture models (Lebreton, Burnham, Clobert and Anderson, 1992; Williams, Nichols and Conroy, 2002). An asset of the Constant Eff ort design is that probabilities of recapture (at a site) can be considered constant over time, simplifying the model structure (Peach, 1993; DeSante et al., 1999). However the temporary presence of transient birds (migrants not resident near the site) needs to be accounted for by explicit probabilities within the analysis, and we now describe a modifi ed approach in detail. Note, on account of the high dispersal of juveniles we restrict the analysis to the capture histories of adults. 
Following Pradel et al. (1997) we assume that at the time of ringing a bird has a probability $\mathrm{T}$ of being a transient. That is, it does not breed at the site and is merely caught in transit so that its eff ective chances of being available for subsequent recapture are assumed to be zero. Pradel et al. (1997) assumed that all birds caught in more than one year were, by virtue of the fact, resident birds (for the duration of the breeding season), but those caught in only a single year were a mixture in unknown proportion of resident birds that evaded future recapture, and transients. The CES design, however, means that we also know how many times within a year any individual was encountered. Given that successive visits are at least 3 days apart, usually more, this enables us to extend Pradel' $s$ et al. model by considering those caught in a single year, but repeatedly, also as residents.

Conceptually we can break the capture histories into 2 segments:

1) From ringing to fi rst recapture which may occur within the year of ringing or in a subsequent year. If never seen again, from ringing to the end of the study.

2) From fi rst recapture onwards, at which point the resident status is confi rmed.

To model the data in the fi rst segment, for a single site, we defi ne:

$\Psi_{\mathrm{a}, \mathrm{t}}-\operatorname{Pr}$ (resident bird alive and present at the site in year $\mathrm{t}$, survives and is present in year $\mathrm{t}+1)$,

c - $\operatorname{Pr}$ (surviving resident bird is recaptured in any given year),

$\varepsilon-\operatorname{Pr}($ resident bird, caught and ringed, is caught only once within its inaugural year),

$\mathbf{f}_{\mathbf{i t}}-\operatorname{Pr}$ (bird ringed in year $\mathbf{i}$ is fi rst recaptured in year $\mathrm{t}$

) ,

$\mathbf{X}_{\mathbf{i}}-\operatorname{Pr}$ (bird ringed in year $\mathbf{i}$ is never seen again).

Permanent emigration away from the site of initial ringing to another breeding site within the UK is accommodated by apparent survival, $\Psi_{\mathrm{a}, \mathrm{t}}$. Note that as a consequence of design $\mathrm{c}$ and $\varepsilon$ are assumed to be time invariant. Further, the assumption that a bird is caught independently at successive within-year visits is questionable, thus $\varepsilon$ and c are estimated freely of one another.

Denoting the number of years of the study by $T$, for $\mathbf{i} \in[1, T]$, we derive 


$$
\begin{array}{rrr}
\mathbf{f}_{\mathrm{it}}=(1-\mathrm{T})(1-\varepsilon) & \mathbf{i}=\mathrm{t} \leq \mathrm{T}, \\
\text { and } \quad \mathbf{f}_{\mathrm{it}}=(1-\mathrm{T}) \varepsilon c \psi_{\mathrm{a}, \mathrm{t}-1}{ }_{\mathrm{k}=\mathrm{i}}^{\mathrm{t}} \overline{\mathbf{n}}_{\mathrm{t}=\mathrm{i}}(1-\mathrm{c}) \Psi_{\mathrm{a}, \mathrm{k}} & \mathbf{i}<\mathrm{t} \leq \mathrm{T},
\end{array}
$$

Letting $F_{i t}$ denote the number of birds ringed in year $\mathrm{i}$ fi rst recaptured in year $\mathrm{t}, \mathrm{t} \geq \mathrm{i}$, and $\mathrm{X}_{\mathrm{i}}$ the number that were never seen again, the histories up until the fi rst recapture can be summarized in a matrix where the rows have independent multinomial distributions with the cell probabilities above. We denote the Multinomial based likelihood by $\mathbf{f}\left(\mathrm{F},\left.\mathbf{X}\right|_{\mathrm{T}}, \varepsilon, \mathrm{C}, \Psi_{\mathrm{a}, 1 \ldots \mathrm{T}-1}\right)$.

Clearly all birds contributing to the second segment are residents and thus a familiar Cormack-Jolly-Seber type model, with temporal survival, is appropriate. The probability that a resident bird recaptured and released in year $\mathrm{i}$ is next recaptured in year $\mathrm{t}, \mathrm{t}>\mathrm{i}$, is:

$$
p_{i t}=c \psi_{a, t-1} \sum_{k=i}^{t}(1-c) \psi_{a, k} .
$$

We let $\mathrm{R}_{\mathrm{it}}$ denote the number of resident birds recaptured and released in year $\mathbf{i}$ that are next recaptured in year $\mathbf{t}$, and $\mathbf{Z}_{\mathbf{i}}$ the number released in year $\mathbf{i}$ that are never seen again. The Multinomial based likelihood is denoted by $f\left(R, Z \mid c, \Psi_{a}, \ldots T-1\right)$.

The model is completed by recognising that we have further information on the parameter $\varepsilon$ arising from multiple within-year encounters from the data in segment 2 . Under the assumption of "constant eff ort" the probability a resident is only caught once within its ringing year, $\varepsilon$, equals the probability a resident is caught exactly once (given that it is caught at all) in any other year. Thus for $t \in[2, T]$ we defi ne:

$\mathrm{M}_{\mathrm{t}}$ - the number of ringed resident birds that are recaptured in year $\mathrm{t}$ exactly once; and $\mathrm{M}_{\mathrm{t}}^{*}$ - the number of ringed resident birds recaptured repeatedly in year $\mathrm{t}$.

Therefore $M_{t} \sim \operatorname{Bin}\left(M_{t}+M_{t}^{*}, \varepsilon\right)$, and the Binomial based likelihood, $\mathbf{f}\left(\mathbf{M} \mid \varepsilon, M+M^{*}\right)$ results. Note that $M_{t}+M_{t}^{*}={ }_{i}{ }_{i} R_{i t}$.

The full likelihood for the capture histories of all birds (at a site) is then given by a combination of the above component likelihoods. A total likelihood for multiple sites follows by assigning each site $g$ its own recapture probability, $c_{g}$, and " evasion" probability, 
$\varepsilon_{g}$, and multiplying the likelihoods for the independent sites. The same apparent survival rates $\psi_{\mathrm{a}, \mathrm{t}}$ are adopted across all sites, as these sites are assumed to sample with varying eff ort the same wider population of birds. If each site were only visited once per year, that is $\varepsilon=1$, our extended model is equivalent to the original Pradel et al. (1997) model.

True survival, $\varphi_{a, t}$, cannot be estimated from the CES live-recapture data alone but by setting

$$
\psi_{\mathrm{a}, \mathrm{t}}=\gamma \varphi_{\mathrm{a}, \mathrm{t}},
$$

where $1-y$ is the probability of permanent emigration, information from CES data can be used to estimate $\varphi_{a, t}$ in the integrated model. Here information pertaining to $y$ arises from $L_{\text {dead }}$ and through the population model (4). The full likelihood, for all $G$ sites, is given by:

$$
\begin{aligned}
L_{\text {live }}= & \mathbf{f}\left(F, X \mid T, \varepsilon_{1 \ldots G}, c_{1 \ldots G}, Y, \varphi_{a, 1 \ldots T-1}\right) \times \mathbf{f}\left(R,\left.Z\right|_{c_{1} \ldots G}, Y, \varphi_{a, 1 \ldots T-1}\right) \\
& \times \mathbf{f}\left(\mathbf{M} \mid \varepsilon_{1 \ldots G}, M+M^{*}\right) .
\end{aligned}
$$

\subsection{Bayesian Model Fitting}

Using Bayesian methodology the integrated model is readily fi tted (King, Gimenez, Morgan and Brooks, 2009). The combination of the underlying abundance and survival models in the fully integrated model is summarised by the Directed Acyclic Graph given in Figure 1.

[Figure 1 about here.]

Vague, independent, priors are specifi ed for all parameters. In particular, for the realvalued parameters in the abundance models, and the log-transformed constant k, we specify a Normal prior with mean 0 and variance 10,000 . For the survival, transience, recapture, evasion and emigration probabilities we assume a Uniform[0,1] prior. We use random walk Metropolis-Hastings updates, with a Normal proposal for real-valued parameters, and a Uniform proposal, appropriately truncated, for the unknown probabilities. Proposal variances and step lengths are tuned a priori. The simulations are run for 200,000 iterations with the fi rst 100,000 iterations discarded as burn-in. Essentially identical posterior estimates are obtained from independent replications with diff erent overdispersed starting points, so that we assume the chain has converged. 


\section{RESULTS}

The analyses below demonstrate that integration of the individual demographic models, $L_{a}, L_{j}, L_{\text {live }}$ and $L_{\text {dead }}$, can lead to a substantial improvement in precision. This is particularly true for juvenile survival in which there is very sparse direct information available. Furthermore, for parameters for which direct information is limited, the underlying demographic model (4) will drive their estimation.

\section{1 “Baseline" Models}

We begin by separately estimating trends in adult abundance, $A_{t}$, and juvenile abundance, $J_{t}$, via models (1) and (2) respectively. These are not restricted by any demographic assumptions, thus they serve as a "baseline" for similar estimates derived under popula- tion models such as (4). Baseline estimates for the productivity indices, $\mathrm{P}_{\mathrm{t}}$, are derived by calculating the ratio of $J_{t}$ to $A_{t}$ using samples drawn from their posterior distributions. As estimates of true survival, $\varphi_{\mathrm{j}, \mathrm{t}}$ and $\varphi_{\mathrm{a}, \mathrm{t}}$ are not obtainable using CES data alone, base- line values are obtained from the ring-recovery data. In addition, a baseline for apparent adult survival, $\psi_{\mathrm{a}, \mathrm{t}}$, is estimated from the CES live-recapture histories via the extended Pradel et al. (1997) model augmented with the Binomial terms as in Section 2.5 .

\subsection{Integrated Model with Ring-Recovery Data}

A wide range of analyses are possible in the integrated context. Initially we form a model by multiplying the likelihoods $L_{a}$ and $L_{j}$ for the adult and juvenile counts from the CES data with the information from the ring-recoveries via $L_{\text {dead }}$. These two data sets are fully independent. Posterior means of $\mathrm{J}_{\mathrm{t}}, \varphi_{\mathrm{a}, \mathrm{t}}, \varphi_{\mathrm{j}, \mathrm{t}}$ and the derived $\mathrm{A}_{\mathrm{t}}$ and $P_{t}$ parameters are shown in Figure 2. Comparisons with the baseline estimates reveal that although the derived adult abundance indices from the integrated model are very similar to their baseline (Figure 2a), the adult survival estimates (Figure 2d) and the juvenile survival estimates (Figure 2e) are quite diff erent. Therefore the adult abundance data, through the recursive equation (4), seems to be driving the estimation of adult and juvenile survival, which is expected as the ring-recovery data are relatively limited. 
Conversely, as the derived adult abundance indices and its baseline are almost exactly the same, this suggests that $L_{\text {dead }}$ has practically no infl uence on the recursive equation.

[Figure 2 about here.]

Figure 2 illustrates the changes in the estimates of adult and/or juvenile survival in the integrated model, not refl ected in their baseline models, to produce derived adult abundance indices that correspond to the adult count data. Note that productivity, and consequently juvenile abundance, is much reduced after 1995, and that since then only three years have been followed by an increase in adult abundance 1998, 1999 and 2003 (Figure 2a). These years (along with the imprecise terminal years) are also those in which adult and juvenile survival is greatly increased under the integrated model. Here, estimates of $\mathrm{J}_{t}$ and $\mathrm{P}_{\mathrm{t}}$ arise from considerably more data than those of $\varphi_{\mathrm{a}, \mathrm{t}}$ and $\varphi_{\mathrm{j}, \mathrm{t}}$, thus the former are more resistant to change once the component models are integrated. $P_{t}$ and/or $J_{t}$ in these three years is not sufficient so the integrated model increases the estimates of $\varphi_{\mathrm{a}, \mathrm{t}}$ and $\varphi_{\mathrm{j}, \mathrm{t}}$ to account for the greater number of adult birds caught.

Integration noticeably improves the precision in the estimates of adult and juvenile survival (Figure $2 \mathrm{~d}, \mathrm{e}$ ). In the baseline $\varphi_{\mathrm{a}, \mathrm{t}}$ and $\varphi_{\mathrm{j}, \mathrm{t}}$ are freely estimated from ring-recovery data unrestricted by any assumptions relating them to adult abundance. In the integrated case, however, the limited information on $\varphi_{a, t}$ and $\varphi_{j, t}$ from the ring-recovery data is augmented by the population model (4) which relates the number of adults in consecutive years to productivity and survival. This extra information improves the precision in the estimates of $\varphi_{\mathrm{a}, \mathrm{t}}$ and $\varphi_{\mathrm{j}, \mathrm{t}}$.

\subsection{Including Capture-Recapture Data}

The much greater amount of adult survival information in the live-recapture likelihood $\mathrm{L}_{\text {live }}$ makes it appealing to further multiply this into the joint likelihood. However, as $L_{\text {live }}$ is drawn from the same data as $L_{a}$ the likelihoods are not independent. Therefore we initially split the data into two groups (one of 35 sites and the other of 36 sites); one is then used to derive $L_{\text {live }}$ and the other independent set to form $L_{a}$. To ensure both 
split data sets provide good coverage over the duration of the study, sites are stratifi ed according to when they joined the CES scheme, and within each of these strata half the sites are randomly assigned to each group. Secondly, we ignore the issue of dependence and derive $L_{a}$ and $L_{\text {live }}$ from the full 71-site data. In both cases $L_{\text {dead }}$ is also incorporated to provide more direct information on $\varphi_{\mathrm{j}, \mathrm{t}}$ so that:

$$
\mathrm{L}_{\text {joint }}=\mathrm{L}_{\mathrm{a}} \times \mathrm{L}_{\mathbf{j}} \times \mathrm{L}_{\text {dead }} \times \mathrm{L}_{\text {live }}
$$

$L_{j}$, which is independent of $L_{a}$ and $L_{\text {live, }}$ is derived from the full 71-site data in each case.

The posterior means of key parameters, $A_{t}, J_{t}, P_{t}, \Psi_{a, t}$ and $\varphi_{j, t}$ from the full and split data analyses are compared to each other, and their baseline estimates, in Figure 3. There are considerably more data on adult survival from the live-recaptures than the limited ring-recoveries, but as the live-recapture data alone cannot provide a baseline for $\varphi_{\mathrm{a}, \mathrm{t}}$, we present estimates of $\psi_{\mathrm{a}, \mathrm{t}}$.

\section{[Figure 3 about here.]}

Concentrating initially on the full 71-site integrated analysis we note that $A_{t}, J_{t}, P_{t}$ and $\Psi_{\mathrm{a}, \mathrm{t}}$ are all very similar to their baseline estimates. Conversely there have been some big adjustments to $\varphi_{\mathrm{j}, \mathrm{t}}$. This is to be expected, since baseline data on $\varphi_{\mathrm{j}, \mathrm{t}}$ is limited the integrated model alters $\varphi_{\mathrm{j}, \mathrm{t}}$ to make the derived $\mathrm{A}_{\mathrm{t}}$ correspond to the adult count data. For example, in Figure 2a the integrated model has explained the increase in adult abundance from 1998, 1999 and 2003 by increasing both adult and juvenile survival (Figures 2d, e), for which little is otherwise known. This does not happen in Figure $3 d$ because the baseline likelihood $L_{\text {live }}$ is now also a part of the integrated model, bringing in substantially more information about adult survival. There is still a paucity of direct information on juvenile survival hence now the integrated model responds to account for the increase in adult abundance by just increasing $\varphi_{\mathrm{j}, \mathrm{t}}$ in these three years (Figure $3 e$ ).

The integrated approach also tends to result in an improved precision. This is most noticeable for juvenile survival (Figure 3e). Here the very limited direct information on $\varphi_{\mathrm{j}, \mathrm{t}}$ from the ring-recovery data is augmented in the integrated model by the information from the deterministic population model, (4). 
The full and split data analyses produce consistent results (Figure 3 ). Using the CES data as two independent groups of 36 sites and 35 sites, we fi nd $A_{t}, P_{t}$ and $\psi_{a, t}$, resemble their baseline, though slightly less so than previously (Figure $3 a, c, d$ ). This is to be expected as fewer data are now used to form $L_{\text {live }}$ and $L_{a}$. For $\varphi_{j, t}$ (Figure 3e) both integrated models produce trends more similar to each other than to the imprecise baseline estimates. As the full data are used to derive $L_{\mathbf{j}}$ in both the integrated models $J_{t}$ is virtually unchanged (Figure $3 b$ ).

For the integrated models, the $95 \%$ symmetric credible intervals for $A_{t}, P_{t}, \psi_{a, t}$, and $\varphi_{\mathrm{j}, \mathrm{t}}$ from the split data analysis are wider, but not dramatically so, than those from the full data analysis (Figure 3a, c, d, e). This is to be expected as these parameters are being estimated from less information when the data are split. However, even under a split data analysis including the CES live-recapture data substantially improves the precision in the estimates of both adult and juvenile survival (Figure $2 d$, e and $3 d, e$ ).

To investigate the sensitivity to the split the analysis is repeated with the data sets used to form $L_{\text {live }}$, or $L_{a}$, swapped over. The trends in the indices between the two split analyses are similar as indicated by the high correlation in their posterior means $\left(A_{t}\right.$ : $\left.\rho=0.86, J_{t}: \rho=1.00, P_{t}: \rho=0.67\right)$. Likewise, the estimates of $\psi_{a, t}$, and $\varphi_{j, t}$ are similar between the two split analyses (see Appendix A).

The proportion of transients $\mathrm{T}$ also requires estimation under the integrated model. The posterior mean (standard deviation) of $\mathrm{T}$ from the full data analysis is $0.37(0.0138)$, and from the two split data analyses is $0.36(0.0207)$ and $0.38(0.0176)$ respectively. The estimate is more precise under the full data analysis due to the greater amount of data.

The integrated model produces estimates of " true seasonal" productivity, the number of young per breeding pair, through the combination of $k$ and $\mathrm{P}_{\mathrm{t}}$, which are consistent with published analyses of nest record data. Assuming an equal proportion of female and male birds, the integrated model estimates, on average, 2.7 young per pair per year. For Sedge Warblers nest failure probabilities, the proportion of nests failing completely due to predation or desertion for example, are approximately 0.015 per day at the egg stage and 0.018 per day at the chick stage (Baillie et al., 2009). As the duration of incubation 
and fl edging are typically $17-19$ and $13-14$ days respectively (Cramp, 1992), this gives a proportion of successful nests of about 0.60 . With an average clutch size of 5 eggs, and typically 1 brood per year (Cramp, 1992), this corresponds to 3.0 young per pair. Though this fi gure ignores the (unknown) number of second broods, the losses of individual chicks up to (and shortly after) fl edging in otherwise successful nests and the uncertainty in the estimates, the correspondence with the integrated model is reassuring.

\subsection{Adult Site-Fidelity}

The posterior mean ( $95 \%$ symmetric credible interval) of $\mathrm{y}$, the probability of not permanently emigrating, from the analysis of the full 71 -site data is $0.82(0.696,0.956)$, and the two split data analyses is $0.89(0.755,0.982)$ and $0.80(0.675,0.951)$ respectively, indicating a high, but importantly not a total, degree of site-fi delity.

A Reversible-jump MCMC algorithm (Green, 1995), used to assess the importance of allowing for permanent emigration, provided some posterior support for the hypothesis that $\mathrm{y}=1$, with a Bayes factor equal to 2.3 (Kass and Raftery, 1995) (see Appendix B).

\section{DISCUSSION}

Previous research has used population modelling to combine data from several longrunning bird monitoring schemes. The earlier approaches, including Peach, Siriwardena and Gregory (1999) and Siriwardena, Freeman and Crick (2001), fi tted population models to indices of abundance derived beforehand from census data. The demographic parameters in their deterministic population models were fi xed at their estimated values derived from nest record and ring-recovery data.

Besbeas et al. (2002) furthered the deterministic approaches above, although their method still modelled an index derived from census data rather than the raw data. They used a state-space model for this index in combination with the usual Multinomial-based models for the ring-recovery data, forming a combined likelihood that integrates both census and ringing information. Brooks et al. (2004) and King et al. (2008) demonstrated how the model could be re-cast in a Bayesian framework. 
Freeman et al. (2007) fi tted demographic population models directly to the raw counts but again fi xing the demographic parameters to previously estimated values. Besbeas and Freeman (2006) essentially combine the methods of Besbeas et al. (2002) and Freeman et al. (2007) thereby correctly accounting for both the correlation and sampling variance in both the demographic parameters and the derived abundance indices.

We provide a new approach to Integrated Population Modelling which combines, for the fi rst time, the Bayesian approach of Brooks et al. (2004) and King et al. (2008), and the fi tting to site-specifi c data arrays of Besbeas and Freeman (2006). The various analyses outlined above use partial census data, i.e. territory counts at a sample of sites, to gain information about abundance, whereas our analysis employs ringing data for this purpose. An advantage of using the CES data is that for certain species, like Sedge Warbler and Reed Warbler (Acrocephalus scirpaceus), which occur in specialised habitats, they provide possibly the best information available with regards to abundance. For the majority of passerines CES live-recaptures also provide better survival estimation than that obtained from ring-recoveries, at least for adult birds. In addition it is perhaps the only practical means of indexing true seasonal productivity (fl edged young per breeding pair per year), rather than fl edged young per breeding attempt as nest record data do.

This paper also introduces a new method for dealing with transients that can be used within, or outside, the integrated context, using the extra information contained within the 12 annual visits. Should only a single visit be made annually, the integrated approach remains viable, and $L_{\text {live }}$ is formed exactly as described by Pradel et al. (1997). Moreover, the extended Pradel et al. (1997) model is not limited to CES data alone but can be applied to other "Robust design" (Pollock, 1982) style data, for example Hines, Kendall and Nichols (2003). By allowing temporal variation in $c$ and $\varepsilon$ it generalises to any two stage capture-recapture study where transients are believed to be present, and inference on the primary sampling period is of interest, providing an e fficient model. Temporal, or site, variation in $\mathrm{T}$ is also possible but not considered here for simplicity.

The integrated approach provides a ready means of assessing the degree of site-fi delity to the breeding grounds of adult birds, or strictly speaking to the area surveyed by the 
mist-net. By utilizing both the ring-recovery data and the deterministic population model, the integrated analysis enables true adult survival, $\varphi_{a}$, to be separated from emigration, $Y$, in the CES live-recapture data.

In general, CES data alone will not enable accurate estimation of juvenile survival, as these birds, unlike adults, are not sufficiently site faithful. By integrating the ringrecovery data, albeit limited, in the integrated models for Sedge Warbler we produce the fi rst precise estimates of juvenile survival for this species with any practical value. Furthermore, being able to estimate the productivity scaling factor, $k$, means that for the fi rst time we can estimate productivity in terms of young per adult, rather than an index merely proportional to it. Typically Sedge Warblers lay one clutch per year, but occasionally two (Alker and Redfern, 1996). The integrated model enables estimation of the true seasonal productivity which includes these multiple attempts, as will be the norm for many other species.

The issue of independence between $L_{\text {live }}$ with $L_{a}$ can be overcome by randomly splitting the CES data, prior to analysis, into two sets at the cost of a small increase of variance. Such an approach will not introduce bias, however the resulting split may be sub-optimal in terms of precision. Currently no eff ort has been made to fi nd the opti- mal split, and further research is needed to develop a more rigorous approach in this respect. Using the full data to derive $L_{\text {live }}$ and $L_{a}$ seems to result in unbiased estimates of the demographic parameters, however variability estimates are likely to be slightly under-reported as a result of, essentially, using the same data twice.

A key advantage to the integrated approach is that it produces estimates of abundance, productivity and survival consistent with both the CES and NRR data sets, and according to the relationship imposed by the population model (4), with one another. Furthermore the estimates are robust across both sources of data. Previous integrated models combine demographic information from entirely separate sources of data, requiring the need to ensure that these relate to the same population. By using CES data to provide direct information on abundance, productivity and adult survival, augmented with additional information on juvenile and adult survival from the NRR database, enables 
more consistency in the sources of information employed.

The integrated approach presented here has a much wider applicability than that of Sedge Warbler CES data alone. Not only does the CES scheme provide sufficient data to monitor other species, but there are many other Constant Eff ort ringing programmes directly modelled on the BTO' S CES scheme in Europe, and the MAPS scheme in the USA, that generate similar data.

The current analysis provides wide scope for future work. Of particular interest is the inclusion of environmental covariates and the investigation of their relationship with the underlying demographic rates, for example that found by Peach et al. (1991) between Sedge Warbler adult survival and Sahel rainfall. Similarly, productivity or juvenile survival may be related to the weather conditions during the British summer. To date we have restricted our analysis to the subset of complete capture records. The ability to improve precision by including additional data for years with missed visits is very appealing, however for these years the assumption of constant eff ort is violated and requires special treatment. Extension to include data with incomplete coverage using the methods of Cave et al. (2008) and Cave et al. (2009) in an integrated context is an area of ongoing research. Further, overdispersion of count data relative to the Poisson distribution is a common problem. Failure to address this leads to an over-reporting of the precision in the parameter estimates. The abundance models for adult and juvenile counts can be readily adapted to incorporate overdispersion, either following the approach of Link and Sauer (2002) or by specifying a Negative Binomial distribution in place of the Poisson.

\section{ACKNOWLEDGEMENTS}

The authors would like to thank the CES ringers for the eff ort they spent collecting the data. The UK ringing scheme is jointly funded by the BTO, the Joint Nature Conservation Committee (on behalf of Natural England, the Countryside Council for Wales, Scottish Natural Heritage, the Environment and Heritage Service of Northern Ireland) and the ringers themselves. The research in this paper was made possible by funding from the National Centre for Statistical Ecology through an Engineering and Physical 
Sciences Research Council grant. Additional funding, for Vanessa Cave, was also generously provided by AgResearch Ltd (New Zealand). We also thank Stephen Baillie, Dawn Balmer, Jacquie Clark, Mark Grantham, Jeremy Greenwood and Rob Robinson of the BTO, Shelley Hinsley of the $\mathrm{CEH}$, and Steve Brooks, for their advice and valuable input regarding this research. Furthermore, we are grateful for the suggestions and comments made by two anonymous referees which have led to a greatly improved manuscript.

\section{APPENDIX A}

Posterior means of $\mathrm{J}_{\mathrm{t}}, \psi_{\mathrm{a}, \mathrm{t}}, \varphi_{\mathrm{j}, \mathrm{t}}$ and the derived $\mathrm{A}_{\mathrm{t}}$ and $\mathrm{P}_{\mathrm{t}}$ parameters from the two split data analyses are shown in Figure 4. The estimates for the indices of adult abundance, juvenile abundance and productivity (Figure $4 a, b, c$ ) are similar between both analyses, as are the estimates of adult and juvenile survival (Figure $4 d, e$ ). The main discrepancy occurs in 2002; under the fi rst split analysis an increase in $\varphi_{\mathrm{j}, \mathrm{t}}$ and a decrease in $\Psi_{\mathrm{a}, \mathrm{t}}$ are estimated, whereas the converse is the case when the data sets are swapped over (Figure $4 d, e$ ). However, the majority of the diff erences are minor, and the analysis seems relatively insensitive to the split.

Interestingly the $95 \%$ symmetric credible intervals of $A_{t}$ and $P_{t}$ from the fi rst split analysis are narrower than those when the data sets are swapped over (Figure $4 a, c$ ). This is because the indices are relative to a reference year (1987) and site. The two split analyses were required to have a diff erent reference site, and this change aff ects the posterior variation of $\beta^{a}$ and consequently the precision of $A_{t}$ and $P_{t}$. Note that posterior variance of $\beta^{a}$ was lower in the fi rst split analysis $(0.0100)$ than in the second (0.0839) resulting in the observed improvement in precision.

[Figure 4 about here.]

\section{APPENDIX B}

The importance of allowing for permanent emigration away from the CES sites in $L_{\text {live }}$ is assessed using a Reversible-jump MCMC algorithm (Green, 1995) that distinguishes 
between the integrated models with and without permanent emigration. Both models are given an equal prior weighting. In the model updating step the probability of proposing a move between models is set to 1 , and normal proposal for $\gamma$, appropriately truncated and tuned a priori, is used. The algorithm is run for 500,000 iterations with the fi rst 300,000 discarded as burn-in, and uses the full 71-site data to derive both $L_{\text {live }}$ and $L_{\mathrm{a}}$. Essentially identical results were obtained from multiple runs with overdispersed starting points, so that we assume the chain has converged. With a Bayes factor equal to 2.3, our results provide weak posterior support that, due to a lack of site-fi delity in adult Sedge Warblers, $\mathrm{y}=1$ (Kass and Raftery, 1995).

\section{REFERENCES}

Alker, P.J. and Redfern, C.P.F. (1996). Double brooding and polygyny in Sedge Warblers Acrocephalus schoenobaenus breeding in north-west England. Bird Study 43, 356-363.

Baillie, S.R. and Green, R.E. (1987). The importance of variation in recovery rates when estimating survival rates from ringing recoveries. Acta Ornithologica 23, 41-60.

Baillie, S.R., Marchant, J.H., Leech, D.I., Joys, A.C., Noble, D.G., Barimore, C., Grantham, M.J., Risely, K. and Robinson, R.A. (2009). Breeding birds in the wider countryside: Their conservation status 2008. BTO Research Report No. 516. BTO, Thetford.

Besbeas, P. and Freeman, S.N. (2006). Methods for joint inference from panel survey and demographic data. Ecology 87, 1138-1145.

Besbeas, P., Freeman, S.N., Morgan, B.J.T. and Catchpole, E.A. (2002). Integrating mark-recapture-recovery and census data to estimate animal abundance and demographic parameters. Biometrics 58, 540-547.

Brooks, S.P., Catchpole, E.A. and Morgan, B.J.T. (2000). Bayesian animal survival estimation. Statistical Science 15, 357-376.

Brooks, S.P., King, R. and Morgan, B.J.T. (2004). A Bayesian approach to combining animal abundance and demographic data. Animal Biodiversity and Conservation 27, 
515-529.

Burnham, K.P. (1990). Survival analysis of recovery data from birds ringed as young: Efficiency of analysis when numbers ringed are not known. The Ring 13, 115-132.

Caswell, H. (2001). Matrix population models: Construction, analysis, and interpretation, 2nd Ed. Sunderland, MA: Sinauer.

Cave, V.M., Freeman, S.N., Brooks, S.P. and King, R. (2008). Survival of Acrocephalus warblers in Britain: Robust models for recapture data with variable capture eff ort. Technical report. CREEM, University of St Andrews.

Cave, V.M., Freeman, S.N., Brooks, S.P., King, R. and Balmer, D.E. (2009). On adjusting for missed visits in the indexing of abundance from "Constant Eff ort" ringing. In Modeling Demographic Processes in Marked Populations (eds. Thomson, D.L., Cooch, E.G. \& Conroy, M.J.). Springer: Environmental and Ecological Statistics 3, 949-963.

Coi ff ait, L., Clark, J.E., Robinson, R.A., Blackburn, J.R., Grantham, M.J., Marchant, J.H., Barber, L., De Palacio, D., Griffin, B.M. and Moss, D. (2008). Bird ringing in Britain and Ireland in 2007. Ringing \& Migration 24, 104-144.

Cramp, S. (ed.) (1992). Handbook of the birds of Europe the Middle East and North Africa: The birds of the Western Palearctic, Volume VI. Oxford University Press.

DeSante, D.F., O' Grady, D.R. and Pyle, P. (1999). Measures of productivity and sur-

vival derived from standardized mist-netting are consistent with observed population changes. Bird Study 46, 178-188.

Fewster, R.M., Buckland, S.T., Siriwardena, G.M., Baillie, S.R. and Wilson, J.D. (2000). Analysis of population trends for farmland birds using generalized additive models. Ecology 81, 1970-1984.

Freeman, S.N. and Crick, H.Q.P. (2003). The decline of the Spotted Flycatcher Muscicapa striata in the UK: An integrated population model. Ibis 145, 400-412.

Freeman, S.N. and Morgan, B.J.T. (1992). A modelling strategy for recovery data from birds ringed as nestlings. Biometrics 48, 217-236. 
Freeman, S.N., Robinson, R.A., Clark, J.A., Griffin, B.M. and Adams, S.Y. (2007). Changing demography and population decline in the Starling Sturnus vulgaris: A multi-site approach to integrated population modelling. Ibis 149, 587-596.

Green, P.J. (1995). Reversible jump Markov Chain Monte Carlo computation and Bayesian model determination. Biometrika 82, 711-732.

Gregory, R.D., Noble, D.G. and Custance, J. (2004). The state of play of farmland birds: population trends and conservation status of lowland farmland birds in the United Kingdom. Ibis 146 (Supplement 2), 1-13.

Hines, J.E., Kendall, W.L. and Nichols, J.D. (2003). On the use of the robust design with transient capture-recapture models. Auk 120, 1151-1158.

Julliard, R., Jiguet, F. and Couvet, D. (2004). Evidence for the impact of global warming on the long-term population dynamics of common birds. Proceedings of the Royal Society: Biological Sciences 271 (Supplement 6), 490-492.

Kass, R.E. and Raftery, A.E. (1995). Bayes factors. Journal of the American Statistical Association 90, 773-795.

King, R., Brooks, S.P., Mazzetta, C., Freeman, S.N. and Morgan, B.J.T. (2008). Identifying and diagnosing population declines: A Bayesian assessment of Lapwings in the UK. Journal of Royal Statistical Society: Series C (Applied Statistics) 57, 609-632.

King, R., Gimenez, O., Morgan, B.J.T. and Brooks, S.P. (2009). Bayesian analysis for population ecology. CRC Press.

Lebreton, J.-D., Burnham, K.P., Clobert, J. and Anderson, D.R. (1992). Modeling survival and testing biological hypotheses using marked animals: A unifi ed approach with case studies. Ecological Monographs 62, 67-118.

Link, W.A., and Sauer, J.R. (2002). A hierarchical analysis of population change with application to Cerulean Warblers. Ecology 83, 2832-2840.

North, P.M. and Morgan, B.J.T. (1979). Modelling Heron survival using weather data. Biometrics 35, 667-681. 
Peach, W.J. (1993). Combining mark-recapture data sets for small passerines. In Marked Individuals in the Study of Bird Population. (eds. Lebreton, J.-D. \& North, P.M.), 107-121. Birkhäuser, Basal, Switzerland.

Peach, W.J., Baillie, S.R. and Balmer, D.E. (1998). Long-term changes in the abundance of passerines in Britain and Ireland as measured by constant eff ort mist-netting. Bird Study $45,257-275$.

Peach, W.J., Baillie, S.R. and Underhill, L.G. (1991). Survival of British Sedge Warblers Acrocephalus schoenobaenus in relation to west African rainfall. Ibis 133, 300-305.

Peach, W.J., Buckland, S.T. and Baillie, S.R. (1996). The use of constant eff ort mistnetting to measure between-year changes in the abundance and productivity of common passerines. Bird Study 43, 142-156.

Peach, W.J., Siriwardena, G.M. and Gregory, R.D. (1999). Long-term changes in overwinter survival rates explain the decline of Reed Buntings Emberiza schoeniclus in Britain. Journal of Applied Ecology 36, 798-811.

Pollock, K.H. (1982). A capture-recapture design robust to unequal probabilities of capture. Journal of Wildlife Management 46, 757-760.

Pradel, R., Hines, J.E., Lebreton, J.-D. and Nichols, J.D. (1997). Capture-recapture survival models taking account of transients. Biometrics 53, 60-72.

Reynolds, T.J., King, R., Harwood, J., Frederiksen, M., Harris, M.P. and Wanless, S. (2009). Integrated data analysis in the presence of emigration and mark loss. Journal of Agricultural, Biological and Environmental Statistics, in press.

Robinson, R.A., Freeman, S.N., Balmer, D.E. and Grantham, M.J. (2007). Cetti' s

Warbler

Cettia cetti : Analysis of an expanding population. Bird Study 54, 230-235.

Sauer, J.R., Hines, J.E., Gough, G., Thomas, I. and Peterjohn, B.G. (1997). The North American Breeding Bird Survey Results and Analysis. Version 96.4. Patuxent Wildlife Research Center, Laurel, MD.

Schaub, M., Gimenez, O., Sierro, A. and Artellaz, R. (2007). Use of integrated modeling to 
enhance estimates of population dynamics obtained from limited data. Conservation Biology 21, 945-955.

Seber, G.A.F. (1971). Estimating age-specifi c survival rates from bird-band returns when the reporting rate is constant. Biometrika 58, 491-7.

Siriwardena, G.M., Baillie, S.R. and Wilson, J.D. (1999). Temporal variation in the annual survival rates of six granivorous birds with contrasting population trends. Ibis 141, 621-636.

Siriwardena, G.M., Freeman, S.N. and Crick, H.Q.P. (2001). The decline of the Bullfi nch Pyrrhula pyrrhula in Britain: Is the mechanism known? Acta Ornithologica 36, 143152.

Underhill, L.G. and Prŷ s-Jones, R. (1994). Index numbers for waterbird populations. I. Review and methodology. Journal of Applied Ecology 31, 463-480.

Van Strien, A.J., Pannekoek, J. and Gibbons, D.W. (2001). Indexing European bird population trends using results of national monitoring schemes: A trial of a new method. Bird Study 48, 200-213.

Vounatsou, P. and Smith, A.F.M. (1995). Bayesian analysis of ring-recovery data via Markov Chain Monte Carlo simulation. Biometrics 51, 687-708.

Wernham, C.V., Toms, M.P., Marchant, J.H., Clark, J.A., Siriwardena, G.M. and Baillie, S.R. (2002). The migration atlas: movements of the birds of Britain and Ireland. T.\& A.D. Poyser, London.

Williams, B.K., Nichols, J.D. and Conroy, M.J. (2002). Analysis and management of animal populations. San Diego: Academic Press. 


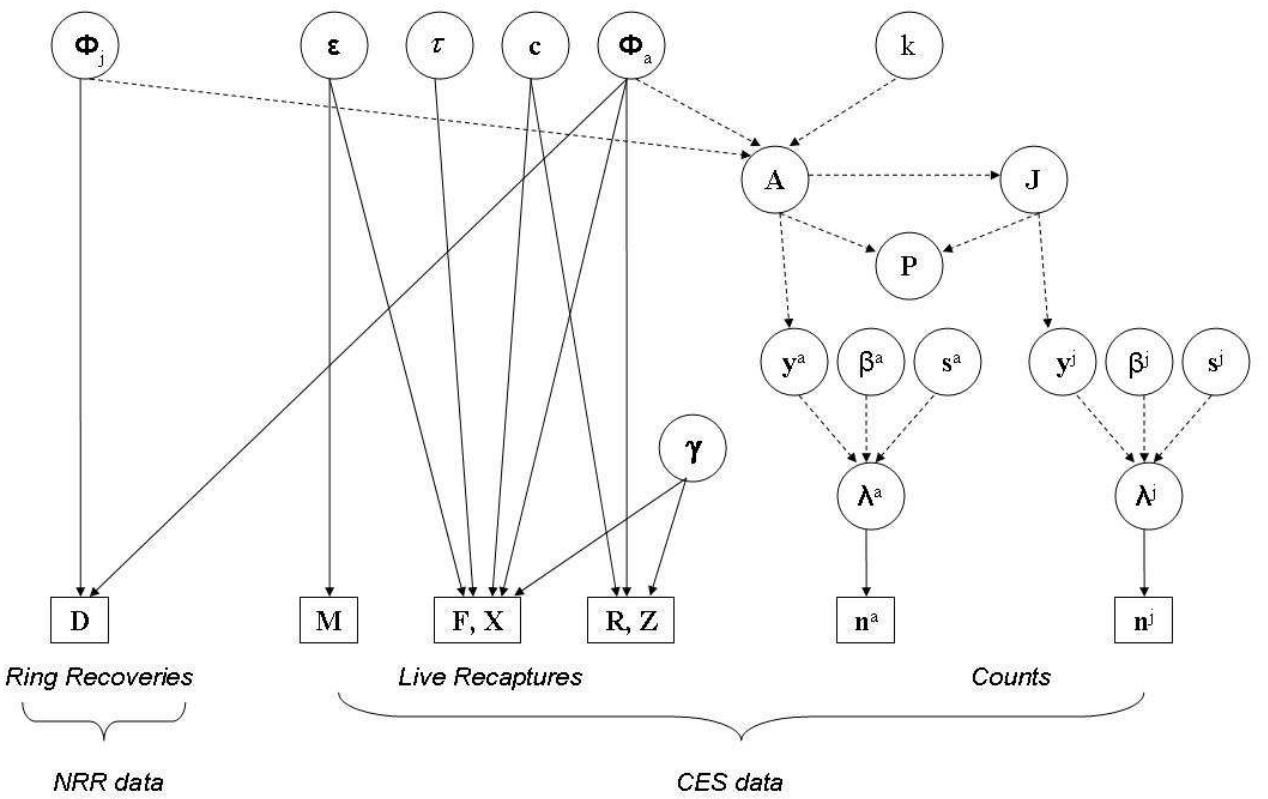

Figure 1. Directed Acyclic Graph corresponding to the fully integrated model. Known values are represented by squares and unknown values by circles. Continuous arrows denote stochastic dependencies and dashed arrows deterministic dependencies. 


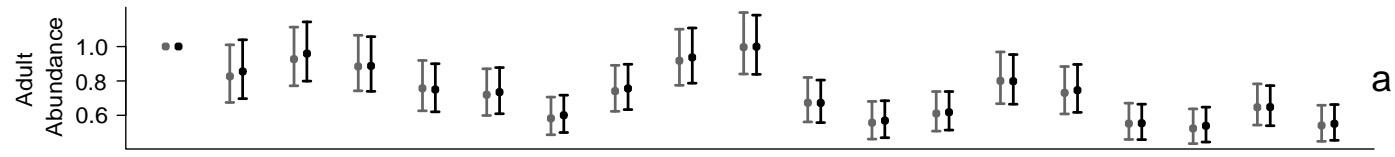

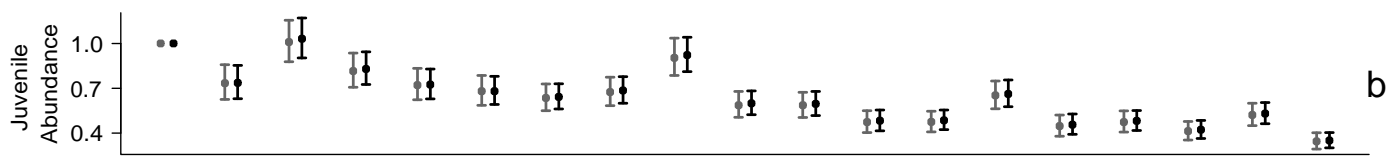

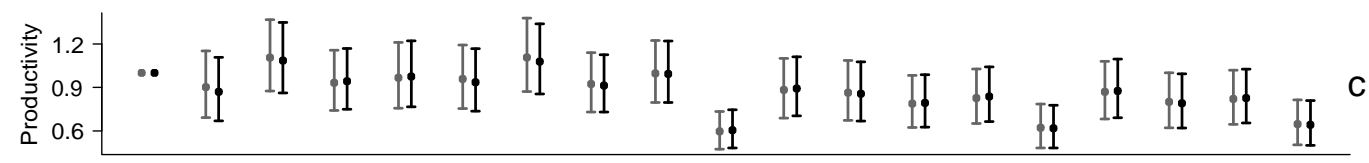

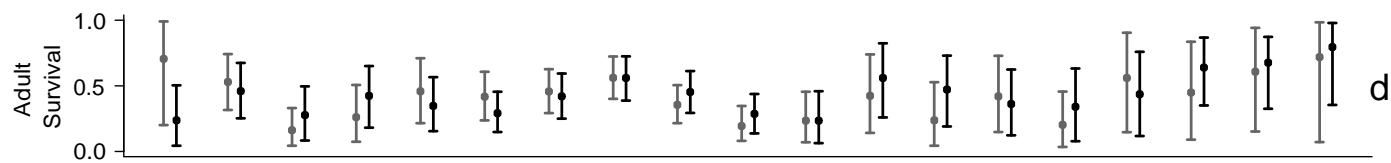

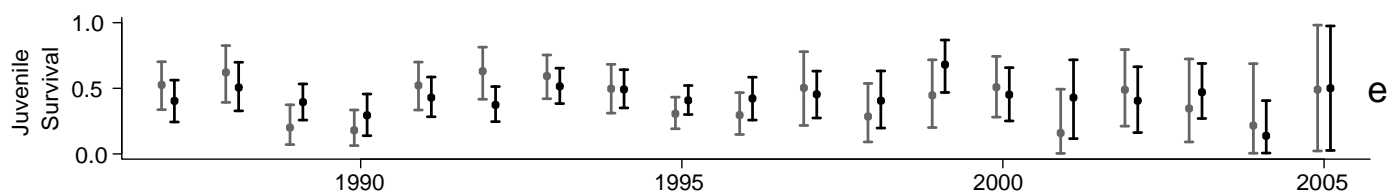

Figure 2. Posterior means, and the $95 \%$ symmetric credible intervals, from the " baseline" models, denoted by left-hand lines, and the integrated model which uses ring- recovery data in the estimation of survival, denoted by right-hand lines, for a) $A_{t}$, b) $\left.J_{t}, c\right) P_{t}$, d) $\varphi_{a, t}$, e) $\varphi_{j, t}$. 


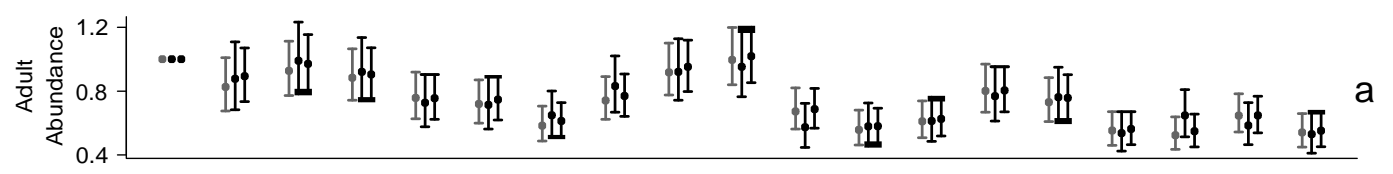

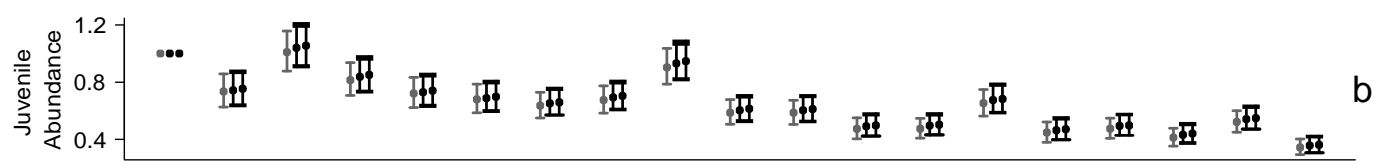

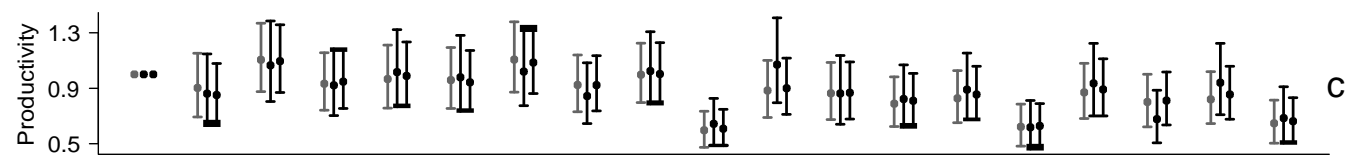

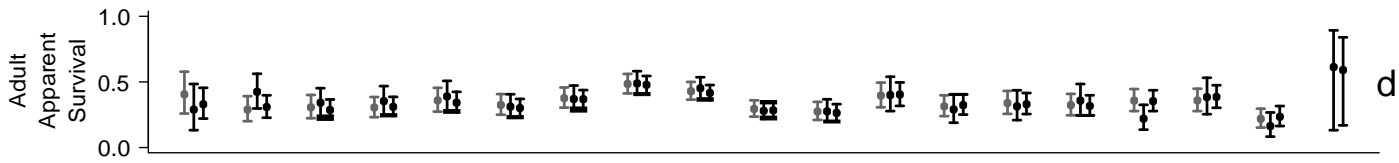

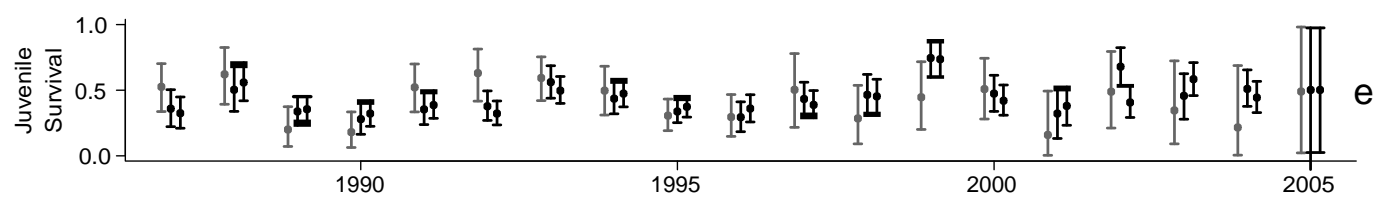

Figure 3. Posterior means, and the $95 \%$ symmetric credible intervals, from the " baseline" models, denoted by left-hand lines, and the integrated model which uses ring- recovery data and CES data in the estimation of adult survival. The analysis in which all 71 sites provide information to $L_{\text {live }}$ and $L_{a}$, is represented by right-hand lines. The centre lines represent the split data analysis. a) $A_{t}$, b) $\left.\left.J_{t}, c\right) P_{t}, d\right) \Psi_{a, t}, e$ ) $\varphi_{\mathrm{j}, \mathrm{t}} \cdot$ 


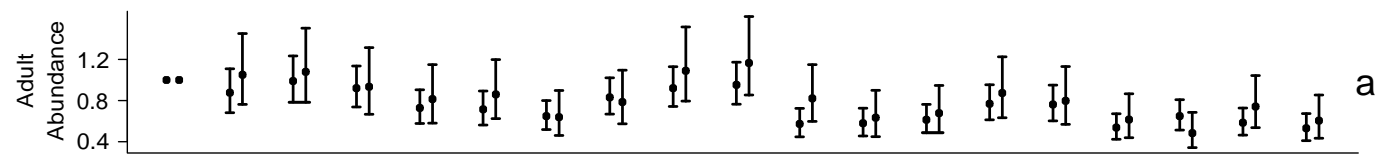

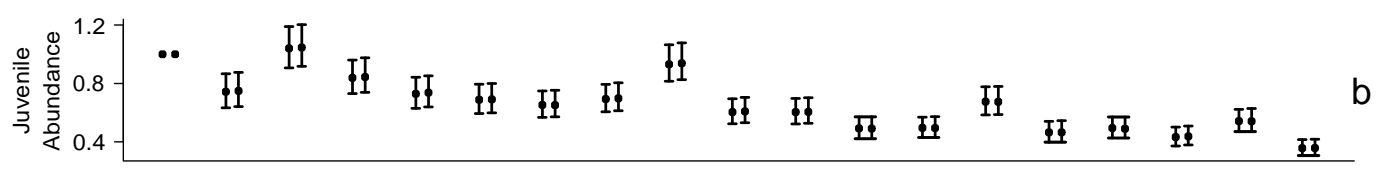

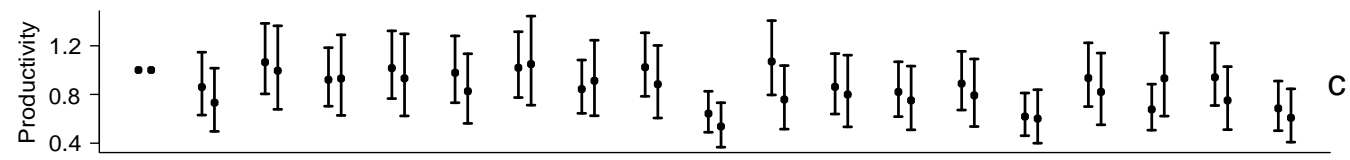

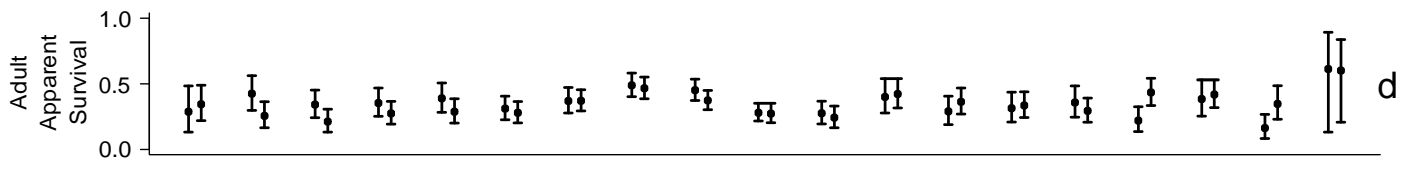

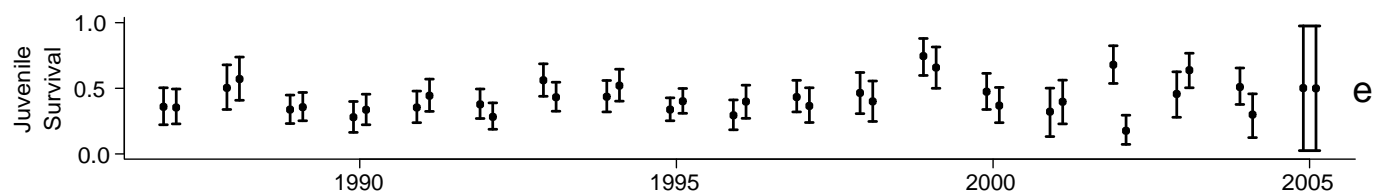

Figure 4. Posterior means, and the $95 \%$ symmetric credible intervals, from the split data analyses where the data are split into two groups; one is then used to derive $L_{\text {live }}$ and the other independent set to form $\mathrm{L}_{\mathrm{a}}$ (left-hand), and then the data sets are swapped over (right-hand), for a) $A_{t}$, b) $\left.J_{t}, c\right) P_{t}, d$ ) $\left.\psi_{a, t}, e\right) \varphi_{j, t}$. 
Table 1. The number of adult and juvenile captures, sites visited and yearly-site counts in the reduced CES data set of " best" sites and the full CES data set of complete capture records from 1987 - 2005.

\begin{tabular}{lrrr}
\hline & Best sites & Full data & Percent \\
\hline Adult captures & 10220 & 13055 & $78 \%$ \\
Juvenile captures & 14627 & 18650 & $78 \%$ \\
Sites & 71 & 192 & $37 \%$ \\
Yearly-site counts & 522 & 767 & $68 \%$ \\
\hline
\end{tabular}

